\title{
Relative occurrence of evidence types in Dutch and French persuasive communication
}

Jos Hornikx (Nijmegen)

\section{Introduction ${ }^{1}$}

Persuasive texts, such as public information brochures or advertisements, aim to convince their readers to behave in a certain manner, such as to stop smoking or to buy a new car. If argumentation is used to achieve this aim, these texts are generally characterised by pragmatic argumentation, a form of argumentation by which an action is recommended on the basis of its favourable consequences. In order to enhance the persuasive effectiveness of these texts, writers may choose to support their claims with different types of evidence, namely statistical, anecdotal, causal, or expert evidence.

A few studies have been conducted to investigate the relative persuasiveness of these evidence types. The question as to what extent these evidence types occur in persuasive communication has hardly been explored. In the present article, a cross-cultural corpus study consisting of Dutch and French persuasive brochures will be presented. Two corpus studies were set up to investigate the relative occurrence of evidence types. Parts of the results have already been presented: study I in Hornikx, Starren and Hoeken (2003), study II in Hornikx (2003). The present article will partly discuss results of these studies, but the most important part will consist of a confrontation of both studies. The studies will be reanalysed with two different statistical methods.

In the following section, the concept of evidence and a rationale for the classification of evidence types will be presented. Subsequently, the two corpus studies will be discussed. The article will end with a general conclusion and a discussion.

1 I am grateful to Hans Hoeken for his comments on a previous version of this article. I would also like to thank him, Marjan Groeneveld and Marianne Starren for their contributions to study II. 


\section{Evidence}

Persuasive texts are written to convince their readers to behave in a certain way. They recommend, for instance, to purchase this type of broadband Internet or they stimulate to stop drinking brandy every day. From an argumentative point of view, these texts can be characterised by pragmatic argumentation, which is also called argumentation on the basis of advantages and disadvantages. Pragmatic argumentation is a form of causal argumentation, in which one reasons from cause to effect (Perelman/ Olbrechts-Tyteca 1969). In its most simple form, the reasoning is as follows: action A leads to consequence $C$; consequence $C$ is (un)desirable; therefore: action $A$ is (un)desirable. In other words, there exists a 'transfer of a given quality from the consequence to the cause' (Perelman/ Olbrechts-Tyteca 1969, 268). Eating fruits and vegetables, for instance, is good, because it reduces the risk of cancer. Pragmatic argumentation can be evaluated on the basis of two main questions (Feteris 2002): one about the normative judgment - is consequence $\mathrm{C}$ desirable? - and one about the empirical judgment - does action A lead to consequence $C$ ? (see figure 1).

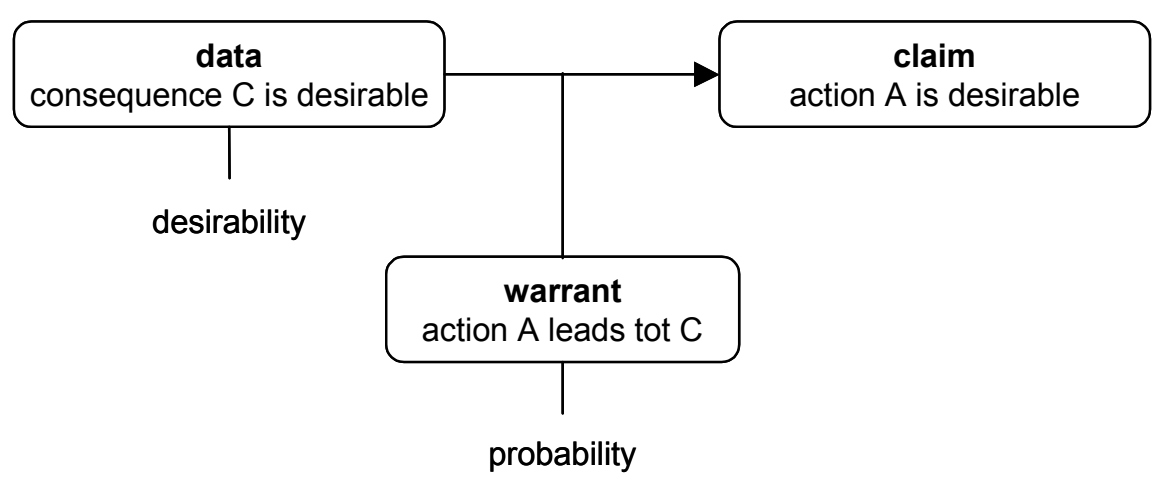

Figure 1: Desirability and probability in pragmatic argumentation

A text writer may anticipate both critical questions of a reader. He can choose to support the desirability of the consequence, and the probability that the action will indeed lead to the consequence. It seems that, in everyday persuasive communication, the desirability of the effects is only rarely supported by evidence. This goes for public discourse (Schellens/ de Jong 2000), and especially for advertising (Schellens/ Verhoeven 1994). The desirability of quick broadband Internet and the undesirability of feeling sick after drinking too much alcohol are self-evident. On the other hand, a reader will not readily accept the implicit claim 
that the broadband Internet of this provider will be very quick or that the consumption of too much brandy will lead to a feeling of sickness. Therefore, evidence can be used to support the probability that an action will lead to certain consequences. Evidence can be defined as "data (facts or opinions) presented as proof for an assertion" (Reynolds/ Reynolds 2002, 429). If a text writer decides to support this probability, he can choose from a large range of evidence types.

There exist many different typologies of evidence types. As Reynolds and Reynolds $(2002,437)$ put it, "Argumentation and persuasion scholars would be well-served by extended efforts at conceptualising and testing different classifications of evidence types". Experimental studies, conducted to measure the relative persuasiveness of evidence types, have generally concentrated on three types of evidence: initially on source evidence (see review of McCroskey, 1969), and later on anecdotal and statistical evidence (see review of Reinard, 1988). There are a few reasons why causal evidence can be added to these three types of evidence. First, causal evidence is one important way by which people may judge the probability of a certain claim. In order to coherently interpret information to assess the likelihood of events or phenomena, people make, amongst others, use of cause-effects relationships (Ajzen 1977, Tversky/ Kahneman 1980). Second, a comparison of the most general argumentation schemes (Garssen 1997) with the three types of evidence leads to the observation that there is no counterpart of the argument by cause in the typology of evidence types. The introduction of causal evidence fills this gap. Third, Hoeken and Hustinx (2002) pointed out that there exists a striking analogy between these four types of evidence on the one hand, and the basic forms of scientific research on the other hand (the experiment, the survey, and the case study). The experiment is conducted to determine possible causal relations between factors, and therefore leads to causal evidence. The survey method is used to question a great number of subjects, and therefore results in statistical evidence. The last method, the case study, is qualitative in the sense that it aims to describe as detailed as possible a case or phenomenon (anecdotal evidence). The only type of evidence lacking in this analogy is expert evidence. There is no research method that leads to expert evidence, but the citation of sources in articles can be interpreted as a form of expert evidence. In order to define the types of evidence, we first have to discuss the function of evidence.

When evidence is used to support a claim, we can speak of an argument. An argument is characterised by its argumentation scheme, the relation between a claim and an argument (evidence in this case). In the argument by generalisation, 
for instance, one reasons that something is the case in general (claim), because it is the case in this specific case (argument). In other words, "what is true of a sample from a class is also true for other members of the class" (Klopf/ McCroskey 1969, 47). Figure 1 shows that, when selecting evidence to support a claim, a warrant, and therefore an argumentation scheme, is generated. A change in the type of evidence will inevitably entail a change in the type of argument. The question then arises why types of evidence are not just types of argument? In support of claims that actions will lead to certain consequences, evidence types are not identical to argument types. One reason is that anecdotal and statistical evidence can each be linked to two different types of argument, depending on the type of claim they support (see Hoeken/ Hornikx 2003). If the claim is general (related to all cases of a given situation), anecdotal evidence leads to the argument by generalisation. The evidence that John felt sick after drinking too much brandy might, for instance, support the claim that, in general, the consumption of too much brandy will lead to a feeling of sickness. If the claim is specific (related to only one case), then the same anecdotal evidence will lead to the argument by comparison (see table 1 below). Here, the same evidence that John felt sick after drinking too much brandy might now support the claim that Harry should not drink too much brandy, because it could make him feel sick.

The definitions of the types of evidence will be given below. They are partly based on the definitions of Rieke and Sillars (1984). We will only concentrate on general claims, because specific claims are usually absent in persuasive communication. Each definition is followed by an example on the basis of the claim that drinking too much brandy will lead to a feeling of sickness.

\begin{tabular}{|l|l|l|}
\hline evidence type & general claim & specific claim \\
\hline statistical & generalisation & Classification \\
\hline anecdotal & generalisation & Comparison \\
\hline causal & causal & Causal \\
\hline expert & authority & Authority \\
\hline
\end{tabular}

Table 1: Evidence types and argumentation schemes per type of claim

Statistical evidence is numerical information, based on a number of cases, that is used in relation to a claim about all the cases of the same class. An example is "Fifteen percent of the people who drink too much brandy experience a feeling of sickness". Anecdotal evidence is the presentation of one case or a few cases that belong to the class mentioned in the claim it supports. An example is "At his 
daughter's birthday party, Johan (53) drank about four glasses of brandy. The day after he felt a bit sick". Causal evidence consists of one or more causes why the event in the claim it is related to will occur. An example of causal evidence is "Alcohol is absorbed into the blood and then affects the organ of balance and the brain". Expert evidence is defined as factual information or opinions that are stated by an expert in relationship to the claim it supports. In this case, evidence could read, "According to alcohol abuse expert Dr. Brown, drinking too much brandy causes a feeling of sickness".

\section{Corpus study}

In the remainder of this article, two corpus studies will be presented. We will first formulate the research questions (3.1) and describe the material (3.2). Subsequently, the procedure (3.3) and the analysis (3.4) will be explained.

\subsection{Research questions}

A number of experiments have been conducted to investigate the relative persuasiveness of evidence types (see for reviews, Baesler/ Burgoon 1994; Reinard 1988). There are hardly any studies that have examined the actual use of these types of evidence in persuasive communication. One of those studies is Levasseur and Dean (1996), in which the relation between the use of evidence in debates between presidential candidates and the outcome of these debates was examined. Based on the data they provide, one can calculate that statistical and anecdotal evidence is more frequently used than source evidence (experts and non-experts). Levasseur and Dean (1996) did not include causal evidence. Above, three reasons were given to add causal evidence to the other types of evidence. Next, studies that investigated the relative persuasiveness of evidence types have failed to examine the role of culture. There are indications, however, that culture may play a role. Nisbett et al. (2001), for instance, gave a detailed overview of differences in reasoning between what they call ancient Greek cultures (e.g., Europe) and ancient Chinese cultures (e.g., China, Japan). There may even exist differences in argumentation within one cultural group. We hypothesise that expert evidence will be more frequently used in French than in Dutch persuasive communication. A text writer who selects expert evidence to support the claim that an action will lead to a certain consequence has a presupposition. That is, in order to be convinced, the reader will have to accept that the expert possesses more 
knowledge about the topic in question. This case is characterised by a high power distance between the reader and the expert (Mulder et al. 1966). The degree to which differences in power distance are accepted proves to be culture-dependent (Hofstede 1991). A high power distance means that people accept the existing power distance; the lower the power distance, the less people accept differences in power distance (Hofstede 1991). France is in general characterised by a high power distance: it scored 68 on a scale of 0 (lowest power distance) to 100 (highest power distance) (Hofstede 1991). People in the Netherlands, on the contrary, generally have a relative low power distance: they scored 38 on the same power distance scale (Hofstede 1991). Therefore, expert evidence might be more frequently employed by French text writers than by Dutch text writers.

Research question 1: is expert evidence more frequently employed in French persuasive communication than in Dutch?

Hornikx et al. (2003) showed that evidence to support the desirability of the consequences is more frequent in brochures with a public interest than in brochures with a direct personal interest. In brochures with a public interest, such as fundraising, a third party experiences the beneficial consequences of the proposed action. In brochures with a personal interest, such as these about alcohol consumption, those consequences are experienced by the reader himself. As both types of brochures differed in the occurrence of support for the desirability of consequences, they may also differ in the relative frequency with which evidence types are used to support the probability that an action leads to (un)favourable consequences.

Research question 2: does reader interest in a brochure have an influence on the relative occurrence of the types of evidence?

\subsection{Material}

In order to test a cultural influence on the relative occurrence of evidence types in persuasive communication, two cross-cultural corpus studies with Dutch and French persuasive public information brochures were set up. Advertisements were not taken into account, because they often lack explicit verbal argumentation. Brochures were selected according to two conditions: they had to be freely 
available for all citizens, and they had to have a persuasive character. We will specify these two conditions below.

In the Netherlands, it was quite easy to collect these brochures, because they are available in all public libraries and town halls. Brochures were selected in the public libraries and town halls of Amsterdam, Den Bosch, Nijmegen, and Veldhoven. In France, public information brochures are mainly distributed by the authorities that publish them; there are only a few institutions that offer a range of different brochures. By far the most important is the French Committee for Health Education (CFES) that belongs to the French Ministry of Health. We contacted several institutions, but only the CFES and the Local Hygiene and Health Service in Rennes sent a number of brochures. We then contacted 45 French fundraising institutions on the basis of an online database (www.yeba.org/annuaire), and an Internet company that groups the most important French fundraising institutions (www.aidez.org).

Not all the collected brochures met the second condition: persuasiveness. A brochure was only considered persuasive, if pragmatic argumentation was the central argumentation scheme. That is, if a brochure presented some kind of behaviour with its (un)favourable consequences, we considered it a persuasive public information brochure, regardless of the style. It was striking how instructive many French brochures proved to be. In these brochures, the accent was not on the consequences of an action, but more on the ways in which the consequences might be achieved: first do this, then do that, etc. The goal of persuasive public information, nevertheless, is the same in the Netherlands as in France. In the Netherlands, this goal is defined as to change the reader's attitude or behaviour in a direction proposed by the text writer (Koelen/ Martijn 1994). In France, the goal is to propose a change of mentality or behaviour that the citizen may adopt (BerthelotGuiet/ Ollivier-Yaniv 2001).

The corpus of study I was collected between October 2001 and May 2002 and consisted of 123 Dutch and 79 French brochures of which only 30 Dutch and 20 French proved to be persuasive. The brochures of the corpus in study II were collected between October 2001 and August 2002 and led to 140 Dutch and 100 French brochures ${ }^{2}$. A number of brochures were also collected in Paris. Study II has two major improvements concerning its corpus. First, the number of brochures per group was kept equal (22 per country). Secondly, the number of brochures per

${ }^{2}$ Both studies have a number of brochures in common. Together the corpora of studies I and II contain 94 brochures; there are 18 brochures that occur in each corpus. 
thematic category was identical over the two countries, because the topic of a brochure might influence the occurrence of certain types of evidence. A text writer of a brochure about road accidents, for instance, might have much statistical information about accidents, but might lack anecdotes. For this reason, the Dutch and the French corpora have the same number of brochures in the eight categories that were found: fundraising, alcohol, traffic, smoking, cancer, health, environment, and Developing Countries (see table 2 below).

These categories were created on the basis of the available brochures. However, the number of available brochures per country varied over some categories. In order to obtain the largest number of brochures, some categories that were finally created are not mutually exclusive. A brochure in the category 'cancer' might of course also fall into the category 'health'. Likewise, a 'fundraising' brochure is often a 'Developing Countries' brochure (but, as in the first example, the opposite is not true). This problem has no further consequences, as the goal of this categorisation is not to compare the relative occurrence of evidence types over the different thematic categories, but to control for the potential influence of the brochures' topic on the occurrence of certain types of evidence in France and the Netherlands.

\begin{tabular}{|l|l|l|l|}
\hline category & Netherlands $(\boldsymbol{n}=\mathbf{2 2})$ & France $(\boldsymbol{n}=\mathbf{2 2})$ & reader interest \\
\hline Developing Countries & 8 & 8 & public \\
\hline fundraising & 5 & 5 & public \\
\hline environment & 1 & 1 & public \\
\hline health & 1 & 1 & personal \\
\hline cancer & 1 & 1 & personal \\
\hline alcohol & 1 & 1 & personal \\
\hline smoking & 2 & 2 & personal \\
\hline traffic & 3 & 3 & personal \\
\hline
\end{tabular}

Table 2: Number of brochures per thematic category in study II $(N=44)$

\subsection{Procedure}

The persuasive information brochures were analysed using the grid of pragmatic argumentation. The procedure consisted of three stages: 1) determining the intended behaviour, 2) searching for the (un)favourable consequences, 3) seeking evidence in support of the probability that the behaviour will lead to the consequences. Only verbal arguments were taken into account, not visual arguments, such as an image of a person. A brochure may recommend one action or a series of actions. Likewise, the number of positive and negative consequences 
may vary from one brochure to another. Each instantiation of evidence was counted as one in the case of expert, anecdotal and statistical evidence. However, when causal evidence consisted of different causes why a consequence would occur, they were together considered as one instantiation of causal evidence. The presupposition here is that causes only form an explanation in combination. Concerning expert evidence, if a person has no superior knowledge regarding the topic, he is not considered an expert, but rather a source. Source evidence (a layman or an expert) can be used in combination with the other types of evidence, like anecdotal or causal evidence. This is even common in everyday persuasive communication: statistical evidence, for example, is "usually provided by reliable sources" (Brosius 2000, 19). Finally, there appear to be two types of expert evidence: an expert designated by name ("According to alcohol abuse expert Dr. Brown"), and an anonymous expert ("Studies show that"). This last category is not taken into account in the present studies, as no difference in power distance can occur: there is a reader on the one hand, but no other person on the other hand.

In study I, the 50 brochures were analysed by one person. After an agreement between three persons about the exact procedure, the reliability was examined by analysing the brochures on two occasions (test-retest reliability). The second analysis only produced four modifications on the total of 127 instantiations of evidence. Subsequently, 14 doubtful cases were discussed by the three persons, which led to only three changes. In contrast to the first study, study II used multiple coders in order to come to a more reliable analysis. One person analysed all the brochures; three other coders each analysed a third of the brochures. The independent analyses of the coders were compared on the basis of two decisions that were made in each brochure: 1) is this really evidence?, 2) which type of evidence is it? Cohen's $\mathrm{k}$ was used to measure the intercoder reliability on both questions: to what degree did the coders agree on their analyses? For both questions, the intercoder reliability proved to be good, as $\mathrm{k}$ was equal or superior to .80 (Neuendorf 2002).

\subsection{Analysis}

In study I, the corpus was analysed with $\chi^{2}$ tests, which is the most common way of statistical analysis in this type of research. The assumption of this method is that the observations (evidence) are independent. As cases of evidence sometimes occur in one single brochure, they may be dependent. A more correct way of 
analysis was used in study II: a 2 (country) x 4 (statistical, anecdotal, causal, expert evidence) analysis of variance. In this case, variance over the brochures is taken into account. Each brochure is considered a subject; country is a between-subjects factor, and type of evidence a within-subjects factor. This method enables one to analyse to what degree the types of evidence are used per brochure per country. Because this method is more conservative, there might be fewer significant results than with $\chi^{2}$ tests. In the present article, the data of studies I and II will be analysed with both methods, as the $\chi^{2}$ test is more common and the repeated measures MANOVA, the more correct one.

In Hornikx et al. (2003), the evidence types in the corpus of study I were classified into the four types of evidence as presented in section 2. When combinations occurred, such as expert evidence with statistical evidence, these were considered both as expert evidence and as statistical evidence. In the current article, as in Hornikx (2002), combinations are seen as independent categories next to the other four types of evidence. In the results that will be presented in the following section, the four pure types of evidence will be emphasised.

\section{Results}

In this section, we will present the results of the analyses of the two corpora. We will first control to what degree both studies are comparable (4.1). We will then present the results according to the two research questions $(4.2-4.3)$. Each time, we will discuss the results of study I ( $\chi^{2}$ tests and analysis of variance), and those of study II ( $\chi^{2}$ tests and analysis of variance).

\subsection{Comparison of study I and study II}

The corpus studies differ on two points: the corpus itself (the brochures in study II are balanced over thematic categories), and the procedure (the brochures in study II are each analysed by two independent coders). The results of study I and study II can be compared. If the occurrences of evidence types in both studies do not significantly differ, this would give more weight to the results. Moreover, this would imply that the analysis of corpus I - which was only analysed by one person - is more reliable than one could think on the basis of the lack of more judges. In fact, the relative occurrence of the four types of evidence in the Dutch and French brochures in study I does not differ from that in study II $\left(\chi^{2}=4.39, d f=7, p=.73\right.$; 
$\left.F(3,90)=1.03, p=.38 ., \eta^{2}=.03\right)$. This shows that the relative occurrence of evidence types is more or less the same over the two corpora. This does not imply, however, that there will be no differences at all in the relative occurrence of evidence types in study I and study II, as we will see in the results. Nevertheless, if we compare the relative occurrences of the four main types of evidence in study I and study II, we can conclude that there are no significant differences between the two studies (see tables 3 and 4 ), which extends the generality of the results.

\subsection{Influence of culture}

The first research question was: is expert evidence more frequently employed in French persuasive communication? An analysis of the corpus of study I shows that expert evidence is used more in the French corpus (see tables 3 and $4 ; \chi^{2}=5.96$, $d f=1, p<.05 ; t(20.97)=1.77, p<.05$; one-tailed). The same results are partly obtained from study II (see tables 3 and $4 ; \chi^{2}=2.95, d f=1, p<.10 ; t(27.16)=1.81$, $p<.05$; one-tailed).

\begin{tabular}{|l|l|l|l|l|l|l|}
\hline & \multicolumn{4}{l}{ study I } & \multicolumn{2}{l|}{ study II } \\
\hline & Dutch & French & total & Dutch & French & total \\
\hline St & 16.13 & $34.43^{* *}$ & 25.20 & 24.14 & 30.23 & 27.78 \\
\hline An & 4.84 & $22.95^{* * *}$ & 13.82 & 6.90 & $29.07^{* * *}$ & 20.14 \\
\hline Ca & $46.77^{* * *}$ & 13.11 & 30.08 & $58.62^{* * *}$ & 22.09 & 36.81 \\
\hline Ex & 1.61 & $9.84^{*}$ & 5.69 & 1.72 & 6.98 & 4.86 \\
\hline Total & 100 & 100 & 100 & 100 & 100 & 100 \\
\hline
\end{tabular}

Table 3: Relative occurrence of evidence types in the Dutch and French brochures (in \% per country; significant differences between countries are indicated; $\mathrm{St}=$ statistical, $\mathrm{An}=$ anecdotal, $\mathrm{Ca}$ = causal, $\mathrm{Ex}=$ expert)

\begin{tabular}{|l|l|l|l|l|l|l|}
\hline \multicolumn{3}{|l}{ study I } & \multicolumn{1}{l|}{ study II } \\
\hline & Dutch & French & total & Dutch & French & total \\
\hline St & $0.33(0.99)$ & $1.05(1.64)$ & $0.62(1.32)$ & $0.64(1.26)$ & $1.18(1.37)$ & $0.91(1.33)$ \\
\hline An & $0.10(0.40)$ & $0.70(1.45)$ & $0.34(1.00)$ & $0.18(0.50)$ & $1.14(2.21)$ & $0.66(1.66)$ \\
\hline Ca & $0.97(1.71)$ & $0.40(0.60)$ & $0.74(1.40)$ & $1.55(1.97)$ & $0.86(1.13)$ & $1.20(1.62)$ \\
\hline Ex & $0.03(0.18)$ & $0.30^{*}(0.66)$ & $0.14(0.45)$ & $0.05(0.21)$ & $0.27^{*}(0.55)$ & $0.16(0.43)$ \\
\hline${ }^{*}<.05$ &
\end{tabular}

Table 4: Occurrence of evidence types per brochure in the Dutch and French corpus (SD between parentheses; significant differences between countries are indicated)

There is also a small category of expert evidence in combination with causal, anecdotal and statistical evidence, which could be called expert+. Expert+ is more frequent in the French corpus with a $\chi^{2}$ test (study I: $\chi^{2}=5.96, d f=1, p<.02$; study 
II: $\chi^{2}=4.77, d f=1, p<.05$ ), but not with a t-test (study I: $t(19.67)=1.05, p=.31$; study II: $t(42)=0.99, p=.33$ ).

More generally, there is a cultural difference in the relative occurrence of the four types of evidence. This goes for study I $\left(\chi^{2}=42.17, d f=3, p<.001 ; F(2.42\right.$, $\left.115.90)=3.37, p<.05, \eta^{2}=.07\right)$, as for study II $\left(\chi^{2}=33.72, d f=3, p<.001 ; F(3\right.$, $\left.40)=2.85, p<.05, \eta^{2}=.18\right)$. When we concentrate on statistical, anecdotal, or causal evidence, there are no cultural differences in the relative occurrence with ttests. However, with a $\chi^{2}$ test, there are significant differences: statistical evidence occurs more often in French corpus (study I: $\chi^{2}=6.64$, $d f=1, p<.01$ ), anecdotal evidence occurs more often in French corpus (study I: $\chi^{2}=11.87, d f=1, p<.001$; study II: $\chi^{2}=12.66, d f=1, p<.001$ ), and causal evidence is more frequently employed in the Dutch corpus (study I: $\chi^{2}=18.83, d f=1, p<.001$; study II: $\chi^{2}=$ 18.80, $d f=1, p<.001)$.

\subsection{Influence of reader interest}

The brochures in the corpora can be divided on the basis of the question whether the consequences of the proposed action will be experienced by the reader of the brochure or by a third party. We can distinguish between brochures with a public interest (such as fundraising and Developing Countries), and brochures with a direct personal interest (e.g., smoking or alcohol). The second research question was: does reader interest in a brochure have an influence on the relative occurrence of the types of evidence? (for the results, see tables 5 and 6). With an analysis of variance, there are no differences in the relative occurrence of the four types of evidence between the two types of interest (study I: $F(3,41)=2.28, p=$ .09; study II: $F(3,38)<1)$. When analysed with a $\chi^{2}$ test, reader interest does have an influence on the relative occurrence of the four evidence types (study I: $\chi^{2}=$ 37.58, $d f=3, p<.001$; study II: $\chi^{2}=13.13, d f=3, p<.01$ ). More specifically, anecdotal evidence is used more in brochures with a public interest (study I: $\chi^{2}=$ 15.73, $d f=1, p<.001$; study II: $\chi^{2}=6.90, d f=1, p<.01$ ), and statistical evidence more in brochures with a personal interest (study I: $\chi^{2}=6.87, d f=1, p<.01$; study II: $\left.\chi^{2}=9.30, d f=1, p<.01\right)$. 


\begin{tabular}{|l|l|l|l|l|l|l|}
\hline \multicolumn{2}{|l}{ study I } & study II \\
\hline & public & personal & total & public & personal & total \\
\hline st & 16.33 & $34.92^{* *}$ & 25.20 & 22.22 & $42.86^{* *}$ & 27.78 \\
\hline an & $26.53^{\star * *}$ & 6.35 & 13.82 & $25.25^{* *}$ & 9.52 & 20.14 \\
\hline ca & 24.49 & 38.10 & 30.08 & 36.36 & 40.48 & 36.81 \\
\hline ex & $14.29^{*}$ & 0.00 & 5.69 & 6.06 & 2.38 & 4.86 \\
\hline total & 100 & 100 & 100 & 100 & 100 & 100 \\
\hline${ }^{*}<.05 ;{ }^{* *}<.01 ;{ }^{* * *}<.001$ &
\end{tabular}

Table 5: Relative occurrence of evidence types in brochures with a public or personal interest (in \% per interest; significant differences between countries are indicated)

\begin{tabular}{|l|l|l|l|l|l|l|}
\hline & \multicolumn{5}{|l|}{ study I } & \multicolumn{2}{l|}{ study II } \\
\hline & public & personal & total & public & personal & total \\
\hline st & $0.36(0.95)$ & $0.96(1.66)$ & $0.62(1.32)$ & $0.76(1.24)$ & $1.38(1.50)$ & $0.91(1.33)$ \\
\hline an & $0.59(1.22)$ & $0.17(0.83)$ & $0.34(1.00)$ & $0.86(1.88)$ & $0.31(1.11)$ & $0.66(1.66)$ \\
\hline ca & $0.55(1.10)$ & $1.04(1.72)$ & $0.74(1.40)$ & $1.24(1.53)$ & $1.31(0.48)$ & $1.20(1.62)$ \\
\hline ex & $0.32(0.65)$ & $0.00(0.00)$ & $0.14(0.45)$ & $0.21(0.49)$ & $0.07(0.28)$ & $0.16(0.43)$ \\
\hline
\end{tabular}

Table 6: Occurrence of evidence types per brochure in brochures with a public or personal interest ( $S D$ between parentheses)

\section{Conclusion and discussion}

Two corpus studies were set up to investigate whether a cultural influence on the relative occurrence of four types of evidence exists: statistical, anecdotal, causal, and expert evidence. Results indeed showed that there is a cultural influence on the relative occurrence of these evidence types (see overview in table 7). The hypothesis that expert evidence would be more frequently employed in French brochures was supported.

The reader interest of a brochure did not affect the relative occurrence of evidence types, when the data were analysed with an analysis of variance. With a different statistical analysis, namely the less correct $\chi^{2}$ test (see section 3.4), reader interest did influence the relative occurrence. Brochures with a public interest, such as fundraising, contained more anecdotal evidence than brochures with a personal interest, which - in turn - had more statistical evidence. There is an explanation for this result. Anecdotal evidence, which is often quite vivid, might be useful in brochures with a public interest, because it enables readers to better understand the consequences of the proposed action. In brochures with a personal interest, a reader will be more able to judge the consequences and the action; in this case, it may be important to show that the consequence has occurred in numerous cases, and not just in one case. 


\begin{tabular}{|l|l|l|l|l|}
\hline & \multicolumn{2}{l|}{$\chi^{2}$} & MANOVA \\
\hline & study I & study II & study I & study II \\
\hline cultural influence? & yes & yes & yes & yes \\
\hline expert more in France? & yes & yes & no & yes \\
\hline influence reader interest? & yes & yes & no & no \\
\hline
\end{tabular}

Table 7: Overview of results depending on the method of analysis and the study

In sum, we can conclude that the two corpus studies show that culture influences the relative occurrence of these types of evidence. This might mean that writers of persuasive texts from different cultures have other intuitions about which types of evidence might be more convincing than others. In order to assess whether these intuitions are correct, experiments should be conducted. In the experiment of Hoeken and Hustinx (2002), for instance, anecdotal evidence had relatively low persuasive power among their Dutch participants, which corresponds with the low occurrence of anecdotal evidence in Dutch brochures in the two corpus studies presented. However, expert evidence was rated as more persuasive than anecdotal evidence (Hoeken/ Hustinx 2002), whereas anecdotal evidence was more frequent than expert evidence in the Dutch corpus. These findings show the relevance of conducting experiments on the relative persuasiveness of types of evidence: evidence types that text writers prefer to use are not necessarily the most persuasive. Experimental research could investigate whether, for instance, Dutch readers appreciate causal evidence more than French readers or whether French respondents find expert evidence more effective than Dutch respondents. In this way, future experimental research could provide more insight into the relative persuasiveness of the different types of evidence.

\section{References}

Ajzen, Icek (1977): Intuitive theories of events and the effects of base rate information on prediction. In: Journal of Personality \& Social Psychology 35-5, 303-314.

Baesler, E. James \& Burgoon, Judee K. (1994): The temporal effects of story and statistical evidence on belief change. In: Communication Research 21, 582-602.

Berthelot-Guiet, Karine \& Ollivier-Yaniv, Caroline (2001): "Tu t'es vu quand t'écoutes l'état": réception des campagnes de communication gouvernementale, appropriation et détournement linguistique des messages. In: Réseaux 19-108, 155-178.

Brosius, Hans-Bernd (2000): Toward an exemplification theory of news effects. In: Document Design 2-1, 18-27.

Feteris, Eveline T. (2002): Pragmatic argumentation in a legal context. In: Frans $H$. van Eemeren (ed.): Advances in Pragma-Dialectics. Amsterdam/Newport News (VA), 243-260. 
Garssen, Bart (1997): Argumentatieschema's in pragma-dialectisch perspectief: een theoretisch en empirisch onderzoek. Amsterdam.

Hoeken, Hans/ Hornikx, Jos (2003): Studying the relative persuasiveness of anecdotal and statistical evidence: What are we comparing? Paper presented at the Southern States Communication Association Conference in Alabama, Georgia, April 3-6, 2003.

Hoeken, Hans/ Hustinx, Lettica (2002): De relatieve overtuigingskracht van anekdotische, statistische, causale en autoriteitsevidentie. In: Tijdschrift voor Taalbeheersing 24-3, 226-236.

Hofstede, Geert (1991): Cultures and organizations: software of the mind. London.

Hornikx, J. (2003). De relatieve frequentie van verschillende evidentietypen in Nederlandse en Franse persuasieve voorlichtingsbrochures. In: L. Van Waes/ P. Cuvelier/ G. Jacobs/ I. De Ridder (eds.), Studies in Taalbeheersing, volume 1 (pp. 206-217). Assen.

Hornikx, J., Starren, M., \& Hoeken, H. (2003). Cultural influence on the relative occurrence of evidence types. In F.H. van Eemeren/ J.A. Blair/ C.A. Willard/ A.F. Snoeck Henkemans (eds.), Proceedings of the fifth conference of the International Society for the Study of Argumentation (pp. 531-536). Amsterdam.

Klopf, Donald William/ McCroskey, James C. (1969): The elements of debate. New York.

Koelen, Maria/ Martijn, Carolien (1994): Persuasieve voorlichting. In: Niels G. Röling/ Dick Kuiper/ Rob Janmaat (eds.): Basisboek voorlichtingskunde. Amsterdam/Meppel, 217-239.

Levasseur, David/ Dean, Kevin W. (1996): The use of evidence in presidential debates: a study of evidence levels and types from 1960 to 1988. In: Argumentation and Advocacy 32-4, 129-142.

McCroskey, James C. (1969): A summary of experimental research on the effects evidence in persuasive communication. In: Quarterly Journal of Speech 55-2, 169-176.

Mulder, Mauk/ Dijk, Rob Van/ Stelwagen, Tilly/ Verhagen, Jan/ Soutendijk, Sibe/ Zwezerijnen, Jan (1966): Illegitimacy of power and positiveness of attitude towards the power person. In: Human Relations 19-1, 21-37.

Neuendorf, Kimberly A. (2002): The content analysis guidebook. Thousand Oaks (CA).

Nisbett, Richard E./ Peng, Kaiping/ Choi, Incheol/ Norenzayan, Ara (2001): Culture and systems of thought: holistic versus analytic cognition. In: Psychological Review 108-2, 291-310.

Perelman, Chaïm/ Olbrechts-Tyteca, Lucie (1969): The new retoric: a treatise on argumentation. Notre Dame/London.

Reinard, John C. (1988): The empirical study of the persuasive effects of evidence: the status after fifty years of research. In: Human Communication Research 15, 3-59.

Reynolds, Rodney A./ Reynolds, J. Lynn (2002): Evidence. In James Price Dillard/ Michael Pfau (eds.): The persuasion handbook: developments in theory and practice. Thousand Oaks (CA), 427-444.

Rieke, Richard D./ Sillars, Malcolm O. (1984): Argumentation and the decision making process $\left(2^{\text {nd }}\right.$ edition). New York.

Schellens, Peter Jan/ Jong, Menno de (2000): Soorten argumenten in de voorlichting. In: Tijdschrift voor Taalbeheersing 22-4, 288-308.

Schellens, Peter Jan/ Verhoeven, Gerard (1994): Argument en tegenargument: een inleiding in de analyse en beoordeling van betogende teksten ( $2^{\text {nd }}$ edition). Groningen.

Tversky, Amos/ Kahneman, Daniel (1980): Causal schemas in judgments under uncertainty. In: Martin Fishbein (ed.), Progress in social psychology. Hillsdale, 49-72. 